\title{
Surface Plasmon Resonance Mode Behaviour in Sierpinski Fractal Triangles and New Plasmonic Materials
}

\author{
Isobel C. Bicket ${ }^{1 *}$, Edson P. Bellido ${ }^{1}$, Danielle McRae ${ }^{2}$, Viktor Kapetanovic ${ }^{1}$, François Lagugné- \\ Labarthet $^{2}$, and Gianluigi A. Botton ${ }^{1 *}$ \\ 1. Department of Materials Science and Engineering, McMaster University, Hamilton, ON, Canada. \\ 2. Department of Chemistry, Western University, London, ON, Canada. \\ * Corresponding authors: bicketic@mcmaster.ca, gbotton@mcmaster.ca
}

Surface plasmon resonances (SPR) are coherent, collective oscillations of conduction electrons confined on the surface of conductive nanostructures. The behaviour of the SPR is determined by the shape, size, and composition of the nanostructure, as well as the surrounding dielectric environment. A given nanostructure may support many different SPRs, each defined by its energy and spatial field distribution. A nanostructure supporting SPR acts as a nano-antenna, interacting with light and paving the way for application of SPRs in areas such as metamaterials [1], sensitive sensors [2], or integrated nano-circuitry [3]. The design of the nanostructure is key to its performance in each application area; we are studying the behaviour of SPR modes in different nanostructures to build the toolbox to enable intelligent design of nanostructures for a given application.

To study the behaviour of SPR in nanostructures, we use a monochromated scanning transmission electron microscope (Thermofisher Scientific Titan at $80 \mathrm{kV}$ ) equipped with an electron energy loss spectrometer, providing both high spatial and energy resolution. Our samples are fabricated on SiN membranes using electron beam lithography. We complement our experiments with finite difference time domain simulations using Lumerical FDTD Solutions.

Fractal geometries, those with a high degree of self-similarity on different length scales, possess a rich complement of SPR modes to study, as demonstrated by Bellido et al. [4]. We are studying the SPR modes present in the Sierpinski fractal triangle. The ideal fractal has infinitely repeating sub-units on all length scales, but fabrication limitations require us to truncate the fractal to a small number of generations (Fig 1a). The self-similarity of the fractal allows us to expand our conclusions from the generations studied to predict the SPR behaviour in higher order fractals. Fractals antennas fill space more efficiently than non-fractal structures, allowing a plasmonic fractal nanostructure to resonate at a longer wavelength and lower energy than a non-fractal structure of the same size. We observe this as a redshift of the dipole mode with successive fractal generations of the same overall size (Fig. 1c).

We show that adding a small hole in the centre of the basic triangle unit to create a generation one (G1) Sierpinski fractal causes a redshift of the dipole peak. Consideration of the Babinet principle [5], [6] reveals that the two degenerate orthogonal dipole modes of the base triangle (Fig 1d, top) couple to two degenerate orthogonal dipole modes on the central hole (Fig 1d, bottom). The larger this hole is made, the stronger the redshift of the dipole peak, until the conductive coupling between the sub-unit triangles in G1 is broken and the SPR response changes to a three-way hybridization of dipole modes of each subunit triangle. The generation two (G2) Sierpinski fractal adds three smaller holes into G1 (Fig. 1a), increasing the redshift seen in the dipole peak for structures of the same size (Fig 1b, c).

With each successive generation, more SPR modes arise from hybridization of the modes of the sub- 
units of the previous generation. Interestingly, the modes retain the self-similarity seen in the different length scales of the fractal (Fig 1b). The number of modes given by hybridization of the dipole modes of previous generations is given by $2 \sum_{n=0}^{N} 3^{n}$, where $N$ is the generation number of the considered fractal, and $n$ iterates over all the previous fractal generations. For fractals of high generation number, there are many different modes possible, making the fractal increasingly broadband with increasing iterations. These features of the Sierpinski fractal geometry make it a good candidate for a space-efficient broadband nano-antenna [7].

Another important factor in the design of plasmonic devices is the material that the device is made of. The traditional noble metals used for plasmonics have high losses, making them challenging to work with for plasmonic circuitry applications. Use of a doped semiconductor material holds promise for low loss circuitry operation; we also show a study of plasmon resonances in indium tin oxide (ITO). ITO is an interesting new plasmonic material compatible with integrated circuit manufacturing processes and supporting infra-red plasmon excitations, with low damping and efficient spatial confinement relative to noble metals making it a good candidate for nano-circuitry applications [8].

\section{References:}

[1] C. Enkrich et al., Phys. Rev. Lett. 95 (2005), p. 203901.

[2] M. Tabatabaei et al., ACS Photonics 2 (2015), p. 752.

[3] V. J. Sorger, et al., MRS Bull. 37 (2012), p. 728.

[4] E. P. Bellido, et al., ACS Nano 11 (2017), p. 11240.

[5] H. G. Booker, J. Inst. Electr. Eng. - Part IIIA Radiolocation 93 (1946), p. 620.

[6] M. Hentschel, et al., Nano Lett. 13 (2013), p. 4428.

[7] I. C. Bicket et al., (2019), Manuscript in preparation.

[8] The experimental work was performed in the Canadian Centre for Electron Microscopy and is supported by the Natural Sciences and Engineering Research Council of Canada (NSERC) under the Discovery Grant Program (ICB, EPB, VK, GAB).
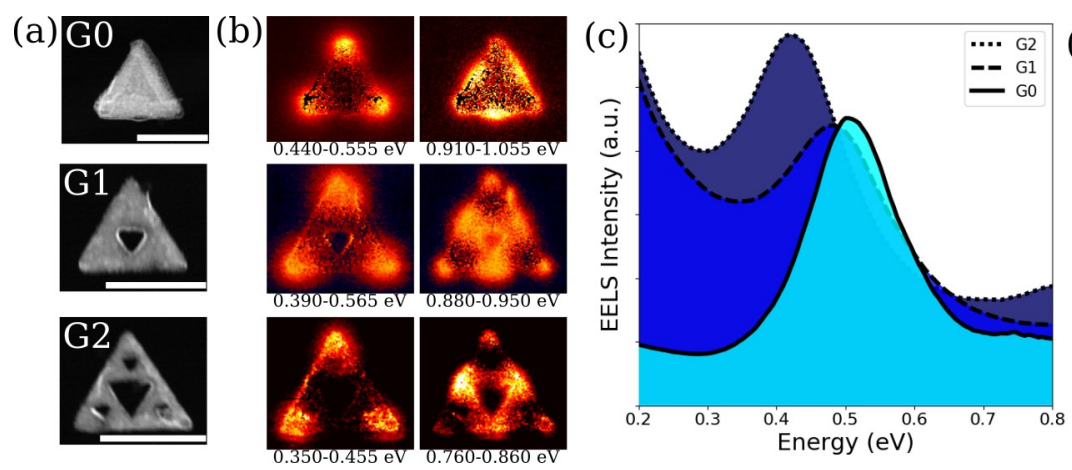

(d)

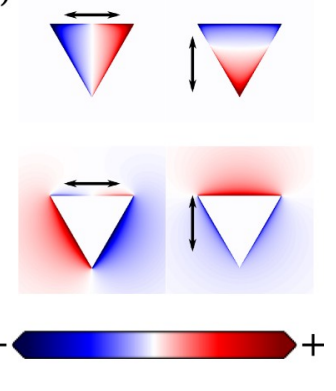

Figure 1. (a) High angle annular dark field images of generation 0 (G0), 1 (G1), and 2 (G2) nanostructures, scalebars are $500 \mathrm{~nm}$. (b) EELS intensity maps for first two SPR peaks for G0, G1, and G2, averaged over the energies indicated. (c) EELS spectra of the dipole modes for G0, G1, G2, averaged over the corners of the triangles shown in (a). (d) Simulated surface charges for the two degenerate dipole modes in a solid triangle (top) and a triangular hole (bottom), photon polarization is indicated by the arrow. 